\title{
Commentaires de cinéma et contrefaçons
}

\section{Movie reviews and pirated posts}

\author{
Bruno Hénocque ${ }^{1}$
}

${ }^{1}$ MCF HC. Université de Normandie (Le Havre). Laboratoire du CIM/CEISME, Labex ICCA, bruno.henocque@bbox.fr

RÉSUMÉ. La promesse d'espaces libres, gratuits, interactifs et démocratiques est indéniablement une donnée historique essentielle du Web 2.0. Les commentaires ou les critiques amateurs en ligne s'inscrivent dans une nouvelle phase du capitalisme que Bernard Stiegler appelle une "économie de la contribution». Cependant, les faux avis (ou faux commentaires) se sont multipliés sur les sites de e-commerce. La question est importante car un seul faux avis peut modifier le choix de l'internaute. Comment dès lors évaluer les commentaires postés sur des sites de cinéma comme AlloCiné ou des sites plus généralistes comme SensCritique ? L'article propose une méthodologie fondée sur l'analyse d'un corpus de commentaires en ligne, suivie d'une analyse sémio-contextuelle de ces commentaires dans le contexte global du Web 2.0 à partir des indices relatifs aux énonciateurs et aux énoncés. La question de l'importance croissante du trafic non-humain généré par des robots est également évoquée.

ABSTRACT. The promise of open, free of charge, interactive and democratic spaces is, without any doubt, an important aspect of Web 2.0. The on-line comments or critics of the non-professionals fall within a new phase of capitalism named Contributive Economy by Bernard Stiegler. Nevertheless, the pirated opinions (or pirated comments) are growing up on the e-commercial sites. The problem is important because at least one pirated comment can modify the choice of the internauts. How can we evaluate correctly the comments posted on the movies' websites, such as Allociné, or on more generic websites, for example, SensCritique (in French: Critical Sense)? This article describes a methodology based on a deep analysis of the on-line comments, followed by the contextual and semantic study in the global context of Web 2.0. The question of a growing importance of the non-human traffic generated by robots is also being studied.

MOTS-CLÉS. Liberté d'expression, Sémio-contextualisation, Typologie des commentaires contrefaits, Typologie des contrefacteurs, Ethique, Contexte juridique, Contexte économique, Relations intersémiotiques entre commentaires, Trafic humain, Trafic non-humain.

KEYWORDS. Freedom of expression, Semio-contextualization, Typology of pirated comments, Typology of counterfeiters, Ethics, Legal and economic contexts, Inter-semiotic relations between comments, Human and non-human traffic.

\section{Introduction}

Pour Bernard Stiegler (2009), nous serions entrés dans une nouvelle phase du capitalisme marquée par l'émergence de l'économie de la contribution. Dans ce cadre, il nous est apparu opportun d'analyser les commentaires amateurs en ligne de films afin de poursuivre nos recherches sur les industries créatives.

Le Web 2.0 se caractérise par la promesse d'espaces publics, gratuits, interactifs et démocratiques de communication. Cette promesse, concernant des sites de commentaires professionnels et amateurs de cinéma, est souvent considérée comme un leurre. Cependant, ces espaces publics ne masquent-ils que les intérêts économiques des industries du cinéma? En adoptant une perspective un peu différente, nous rappellerons que le processus d'appropriation de toute innovation passe par des étapes ou des moments (Hénocque, 2002, pp 89-99). Les sites de commentaires cinématographiques, qui croisent les intérêts des industries du cinéma et de cinéphiles amateurs passionnés, n'échappent pas à leur tour à de sérieuses questions concernant l'augmentation des faux avis. Il suffit en effet parfois d'une seule contrefaçon pour fausser le jugement du cinéphile. Plus généralement, cette question de la contrefaçon est importante car la crédibilité du Web 2.0 (Cardon, 2010) peut être remise en cause.

Or, sur cette question des contrefaçons, le rapport annuel de 2013 de la Direction Générale de la Concurrence, de la Consommation et de la Répression des Fraudes (DGCCRF) présente un bilan un peu inquiétant: le taux de faux avis, sur l'ensemble des sites de e commerce, avait atteint $44,4 \%$. Ce 
chiffre élevé s'applique-t-il vraiment aux sites de commentaires en ligne de films ? Le cinéma n'est pas en effet une activité commerciale comme une autre. Ces sites rassemblent de nombreux passionnés désintéressés. Nous partirons de l'hypothèse prudente d'une large incertitude quant à la fiabilité des contenus mis en ligne sur les sites de commentaires de cinéma, ce qui soulève de sérieuses questions éthiques.

Les commentaires de cinéma sont, d'un point de vue sémiologique, des discours qui font appel au jugement de l'énonciateur. Certes, faits et commentaires s'interpénètrent à des degrés divers dans tous les discours ou les illustrations. Une photographie de presse par exemple, dont le rapport analogique avec le réel peut créer une illusion de vérité, est en fait « un objet travaillé, choisi, composé, construit, traité selon des normes professionnelles, esthétiques ou idéologiques» (Barthes, p 12). La promesse inhérente à ces commentaires (ou " critiques » ou encore " avis ») est d'appartenir à la catégorie des genres discursifs à forte dominante subjective, au même titre que les éditoriaux, chroniques, billets ou dessins de presse. La forme simple et la plus fréquente d'un avis oppose «j'aime» et «j'aime pas ». Olivier Le Deuff (2012) a bien noté que cette opposition est aussi celle du 0 et du 1, base du codage informatique qui est binaire.

Afin d'affiner la compréhension de ce phénomène social, le recours à un corpus de commentaires en ligne de films peut être pertinent, mais comment procéder? Nous avons choisi d'extraire du Web une série de commentaires sur le film biographique Jobs, sorti sur les écrans français en août 2013. Ce film de Joshua Michael Stern était très attendu car ce fut le premier biopic de Steve Jobs, cofondateur d'Apple. Nous avons travaillé sur un échantillon diversifié de commentaires amateurs tirés d'Allociné et de SensCritique afin de faire apparaitre des écarts et de commencer à réfléchir à une typologie. Cette extraction a été effectuée en janvier 2015. Nous avons opté pour un traitement au cas par cas plutôt qu'un traitement linguistique automatique. La condamnation à 500. 000 euros de Tripadvisor par un tribunal italien en 2014 démontre en effet que les logiciels de détection des faux avis ne sont pas infaillibles.

\section{Méthodologie de travail : commentaires et contextes numériques}

\subsection{Approche sémio-pragmatique}

Dans une optique sémio-pragmatique (Odin, 2011), les commentaires sur un site web mobilisent des compétences sémio-linguistiques (maîtrise des aspects du langage), discursives (fonctionnement de ce type de discours), socio-culturelles (codes partagés pour interagir) et référentielles (relations énonciatives).

Une première observation de quelques commentaires nous a permis cependant de déceler la complexité à distinguer les vrais des faux commentaires à partir de ces catégories. Certes, la présence parmi les commentaires conversationnels amateurs, de productions discursives relevant de genres aussi codifiés que l'annonce publicitaire ou le communiqué de presse parait relever d'une pratique trompeuse. Mais sur les indices suivants, il existe une grande incertitude :

- L'étendue et la richesse des références précises à l'œuvre peut être le signe que le commentateur a vu le film, mais rien ne garantit que son commentaire ne soit pas une contrefaçon!

- La fréquence des écarts sémio-linguistiques, discursifs ou référentiels dans les écrits conversationnels peut être la marque d'un énoncé authentique ou celle d'un parfait contrefacteur !

Une approche centrée seulement sur les contenus pourrait donc conduire à bien des conclusions erronées. Il convient, pour ce qui nous concerne, d'analyser à la fois les commentaires en tant que tels et d'autres dimensions constitutives, en interrogeant notamment la complexité du dispositif sociotechnique et en situant ces commentaires dans leur environnement économique et juridique. La recherche dans le dispositif socio-technique permet par exemple de déceler la présence des mêmes formes sémiotiques (textes, images, vidéos) sur d'autres sites Web comme des sites de e commerce, ou 
les mêmes énonciateurs sur des blogs de cinéphiles ou encore les mêmes images libres de droits ou bien piratées.

\subsection{Le dispositif socio-technique}

Pour Edgar Morin (2004),le paradigme de la complexité est le seul paradigme qui puisse rendre compte de la complexité des systèmes et permette de concevoir, d'un point de vue éthique, la science comme à la fois bonne ou mauvaise, bienfaisante et pervers, utile et néfaste. Les moyens complexes de diffusion doivent entrer dans l'analyse des commentaires. Il faut bien en passer par là et revenir pour commencer à l'approche de base de Laswell (1948) qui pose la question de savoir par quels moyens (par quels médias) s'effectue la communication et avec quels effets. Un autre détour est nécessaire par la théorie de la communication éditée en 1949 et complétée dans les années suivantes par Wiener. Weaver souligne avec force que la couche technique a une incidence sur la couche sémantique qui ellemême à une incidence sur l'influence exercée par le message sur le récepteur.

Ainsi, un dispositif de commentaires de film est un espace scripto-visuel public de communication qui repose sur une structure et des éléments récurrents: synopsis, bande-annonce, lien pour accéder au film en VO, présentation des acteurs, interviews, rubriques des commentaires professionnels et amateurs qui forment une communauté discursive. Mais les frontières d'un commentaire en ligne ne s'arrêtent pas au seul écran. Tout commentaire s'inscrit dans un dispositif socio-technique dynamique composé de plusieurs strates, en perpétuelle construction, reliées à de multiples interfaces et logiciels. Une plate-forme de commentaires de cinéma est donc constituée d'un support d'inscription où s'affichent des commentaires et d'un support matériel, relié à la profondeur du web, qui interconnecte des sites, des moteurs de recherche, des interfaces, des logiciels et des routeurs.

Le support matériel conditionne fortement le support d'inscription. En effet, sur le support d'inscription, la place des commentaires est éphémère et leur place bouge en fonction des votes et du classement modifiable des commentaires Pour Edgar Morin et Jean - Louis Lemoigne $(1990,1999)$, chaque objet à décrire est à replacer dans son contexte, afin d'établir les « intercommunications », la « systématicité » et la «multidimensionnalité » des phénomènes. Il ne faudrait pas morceler les objets en les coupant « de leur contexte, de leur localité, de leur singularité, de leur temporalité... ». Les sites Web, les listes de diffusion, les forums, les blogs et les réseaux sociaux développent ainsi entre eux des relations inter-sémiotiques. De multiples liens d'intertextualité relient ainsi un commentaire ou un vote à d'autres commentaires ou à d'autres votes sur la toile. Sans entrer dans les détails, le dispositif global comporte sept couches hiérarchisées selon le modèle OSI (Open Systems Interconnection), modèle qui permet la communication entre systèmes informatiques. Les quatre premières couches concernent la transmission et les trois couches suivantes le traitement de l'information. De même que des fraudes sont possibles dans un service postal (aux étapes du tri du courrier, du routage dans les centres, du libellé des enveloppes ou des conventions de présentation) de même des fraudes peuvent exister dans le cadre d'une interconnexion informatique de systèmes ouverts, par exemple sur le plan de la couche réseau, de la couche transport ou de la couche applicative. Toutefois, Il faut admettre que cette approche globale se heurte à une difficulté majeure : en pratique, la majorité des internautes n'a aucune visibilité sur l'architecture du réseau. Un internaute non spécialiste ne maitrise que quelques données à caractère personnel : son login, son mot de passe, la clé de chiffrement à 12 signes. Il peut accéder aux espaces publics de communication mais n'a pas les droits pour en modifier les contenus. Pour l'essentiel, il doit faire confiance aux FAI et aux entreprises d'architecture de réseau.

De plus, ce dispositif socio-technique s'insère dans un contexte socio-économique considéré comme déterminant pour une longue tradition de recherche en sciences de l'information et de la communication. 


\subsection{Commentaires et contexte socio-économique}

Nous sommes en accord avec François Jost (2010) pour considérer que l'analyse des contenus numériques, en particulier des contenus des sites, doit prendre en compte le contexte économique. Dès l'origine, la publication de commentaires sur les médias sociaux, les listes de diffusion, les forums, les blogs et les sites participatifs a reposé sur des logiques de gratuité et d'interactivité. Parmi les nombreux sites impliquant des communautés discursives, Agoravox est considéré comme le premier site participatif et citoyen en langue française et Rue89, le premier site d'information français à trois voix (journalistes, experts et amateurs), apparus respectivement en 2005 puis en 2007. Le Web 2.0 offre la possibilité « aux critiques -consommateurs de prendre la parole plus facilement, de faire des commentaires sur les produits ou une entreprise » (Agathe Lepage.2006). Tout internaute devient un critique potentiel.

Très vite, s'est alors posée la question du financement des sites par la publicité, Google se rémunérant par les liens hypertextes qu'il vend tout en proposant aussi un référencement payant (Olivier Andrieu, 2015, p437). Dans ce contexte, les marques essaient aussi d'attirer les internautes, en particulier les plus influents, par des cadeaux. Un commentaire favorable d'un e leader donne au produit toutes les chances de devenir un succès. Plus généralement, ces stratégies s'intègrent dans une logique marketing de crossmedia.

Les sociétés de production de cinéma ont elles aussi intérêt à ce que les commentaires des films qu'elles distribuent leur soient favorables. Ce lien étroit entre ressources publicitaires et édition n'est évidemment pas nouveau et apparait dès le premier support de presse français en 1836. Dans le journal d'Emilie de Girardin, la publicité aide à la publication des romans-feuilletons, notamment de Balzac.

Mais si pour subventionner l'édition, la publicité est la seule alternative pour les gestionnaires de sites, elle ne doit pas porter atteinte à l'éthique. Or, la publicité peut être discrètement dissimulée afin de mieux influencer les internautes. Plusieurs affaires de publicités déguisées ont ainsi défrayé la chronique depuis le début des années 2000 avec le développement du Web 2. 0 puis des médias sociaux. Pour des sites Web, l'attachement à l'éthique peut paraitre dérisoire face aux impératifs de rentabilité des gestionnaires des plateformes numériques. De plus, l'existence de pratiques déloyales reste difficile à démontrer en dehors des contrôles ponctuels exercés par la DGCCRF.

La question des faux commentaires est cependant loin d'être anodine. La frontière entre contenus non publicitaires et annonces publicitaires est de plus en plus ténue en raison de la création de sites publicitaires reposant sur des solutions Google comme Adsense. Ces solutions permettent de concevoir des sites gérant à la fois des contenus publicitaires et non publicitaires. La frontière entre les deux domaines d'activités est donc de plus en plus fragile, ce qui soulève des problèmes éthiques et déontologiques car ces deux types de contenus sont normalement séparés. En brouillant les frontières entre commentaires professionnels et publicité, les plates formes de commentaires risquent de fausser l'appréciation puis la décision du cinéphile. En ignorant les intentions du rédacteur, le cinéphile est ainsi manipulé. Jean-Pierre Teyssier (p101) développe un avis très tranché sur cette question : «En définitive, le pire mensonge que puisse commettre la publicité, c'est de ne pas se présenter comme telle ». C'est, explique -t-il, en reconnaissant les codes stylistiques de la publicité que le consommateur peut faire des choix éclairés alors qu'en masquant les intentions publicitaires d'un message, on détruit la distance que le consommateur prend par rapport au message.

Fait plutôt rare, le site www.premiere.fr, spécialisé dans l'actualité du cinéma, des séries TV et des stars, s'est livré à une analyse intéressante des faux commentaires de cinéma sur l'URL www.premiere.fr/cinema/News-cinema/twilight-hunger-games-blanche-neige-le-marketing-va-t-il trop-loin 24/11/2011.

Les commentaires sont par ailleurs reliés à des votes, positifs ou négatifs. En votant de nombreuses fois, l'internaute peut aider un film à figurer parmi les films les mieux notés sur les sites de cinéma. $A$ 
contrario, les « haters » font baisser une note ou un classement, en postant de mauvaises notes et des commentaires négatifs. Le classement en tête d'un commentaire dans une liste dépend du nombre de votes positifs postés. En raison de l'influence exercée par ces votes, l'échange ou l'achat de likes ou de followers est devenu une pratique usuelle sur des sites spécialisés comme www.LikeUp.fr, www.comenlike.com ou www.tooply.com. L'internaute peut aussi s'inscrire sur un site spécialisé et poster des likes sur les pages Web d'un commentateur de cinéma, en devenir «fan» et réciproquement. Des avis avec de nombreux likes ont plus de chances d'être lus. Il est possible ainsi de créer le « buzz » sur les pages d'un contributeur et de « booster » sa popularité.

Une double question se pose également concernant la mise en exergue de certains commentaires. Il faudrait davantage de transparence sur les choix opérés par le gestionnaire (ou le modérateur) du site qui met ou remet en ligne certaines critiques dans les rubriques dédiées alors que des milliers de critiques sont postés tous les mois? Sur quels critères par ailleurs est choisi le «commentaire de la semaine »? Un exemple parmi des centaines d'autres permet de mieux comprendre les enjeux éthiques de tout classement. Le film Conjuring : les dossiers Warren est sorti en salle le 21 août 2013 et a été replacé à la Une du site Allociné le 21 janvier 2015. Le film n'était pourtant plus à l'affiche dans les cinémas UGC du 21 au 28 janvier 2015. En fait, l'explication relève probablement de la chronologie des médias : il fallait relancer l'intérêt pour un film qui faisait alors l'objet d'une rediffusion en télévision payante de cinéma. Par contre, il est difficile d'un point de vue éthique de trouver une logique au classement des 1384 commentaires amateurs écrits sur ce film à cette époque. Sur quels critères a été choisi par exemple comme "commentaire de la semaine» un avis plutôt quelconque posté le 8 mai 2014 ?

Mais il y a plus, beaucoup plus : le trafic non-humain d'avis ou de votes est devenu plus important que le trafic humain. Le trafic non -humain désigne les visites sur les sites et les pages vues qui sont générées par des robots. D'après le rapport annuel de la société de sécurité Imperva Incapsula (2016), la part de ce trafic pour l'ensemble des publicités en circulation sur le Web représenterait $52 \%$ de l'ensemble des publicités, supplantant ainsi le trafic humain. Cette étude impressionnante a porté sur 16.7 billions de visites et 100.000 noms de domaines choisis au hasard du 9 août au 6 novembre 2016 . La question éthique de la visibilité de ce trafic se trouve posée avec force actuellement car il faudrait pouvoir distinguer les différentes catégories de messages. Certains robots (ou bots) sont en effet légitimes : indexation de moteurs de recherche, collecte de données à des fins commerciales...D'autres constituent des spams (près de $80 \%$ du trafic quotidien sur la toile), des actions de piratage de données, des faux comptes et des cas d'usurpation d'identité numérique. Ces bots peuvent simuler des clics et tenter de fausser le lancement d'un film. Ces programmes peuvent permettre au contraire d'éviter aussi que les avis négatifs n'arrivent en tête de liste dans les moteurs de recherche, en cliquant de très nombreuses fois sur le bouton like. Dans l'édition de contenus digitaux, les messages illicites générés par des robots représenteraient actuellement un tiers du trafic global d'après la société Imperva Incapsula. Ces pratiques illicites provoquent non seulement des protestations éthiques mais aussi des réactions juridiques.

\subsection{Commentaires et contexte socio-juridique}

La multiplication de faux avis et de votes générés par des robots visent à tromper et à manipuler les consommateurs. Beaucoup d'usages, en particulier dans les nouveaux médias, témoignent d'un écart important entre la normativité juridique et l'effectivité du droit (Calafat, Fossier, Thévenin, p.7). Peu de commentaires frauduleux débouchent sur un procès car il faudrait apporter les preuves de leur existence, dresser le cas échéant un constat d'huissier, imprimer les commentaires incriminés. Si l'administrateur du site et le FAI disposent des données permettant de contrôler et d'identifier les auteurs de contrefaçons, certaines identifications sont rendues difficiles en raison du brouillage des adresses IP ou bien de la création de faux comptes sur des sites communautaires. A ce titre, Facebook bloque depuis mi 2013 les flux de messages publicitaires frauduleux générés par le logiciel de 
navigation Tor, qui permet d'accéder à des sites non référencés sur le Web et de conserver l'anonymat lors de ses connexions.

Il semble que les sites de cinéma soient moins concernés en Europe par les poursuites judiciaires, que les sites de voyages, d'hôtels, d'automobiles ou de nouvelles technologies. Parmi les cas les plus récents qui ne concernent pas l'industrie cinématographique, citons en premier lieu la condamnation du site français TripAdvisor par un tribunal italien le 22 décembre 2014 à une amende de 500.000 euros pour faux commentaires, pratiques déloyales et manœuvres frauduleuses. Le site diffusait des commentaires de professionnels, en laissant accroire qu'il s'agissait de commentaires de touristes. En 2013, les responsables de ce site avaient reconnu que le traitement automatique des données sur des critères linguistiques, afin de discerner les faux commentaires, conduisait à un taux d'erreurs de $60 \%$ environ!

Le TGI de Paris a par ailleurs condamné le 20 juin 2014 un guide de bonnes adresses à 7000 euros d'amendes pour de faux avis de consommateurs, rédigés par une société étrangère, l'entourage du gérant du site et le gérant lui-même.

Les formes sémiologiques utilisées par les contrefacteurs donnent souvent l'illusion de commentaires authentiques. Les techniques utilisées, d'après la Direction Générale de la Concurrence, de la Consommation et de la Répression des Fraudes (DGCCRF), relèvent de pratiques commerciales trompeuses, difficiles à détecter par un internaute. D'un point de vue juridique, il ne s'agit pas de correspondances privées.

La DGCCRF veille au sein du ministère de l'Economie au respect des règles de la concurrence - y compris sur les sites Web- en luttant entre autres contre les contrefaçons. Elle veille au respect des règles relatives à l'information des consommateurs et à la loyauté des pratiques commerciales, en tentant de détecter les pratiques illicites.

On peut, en s'inspirant de la liste des contrefaçons éditée par la DGCCRF, dresser une typologie plus simple des feintises utilisées en dégageant quatre grandes catégories.

\subsubsection{L'astrosurfing qui consiste à inonder des sites de faux commentaires sur un objet.}

La rédaction d'un très grand nombre d'avis est assurée par un prestataire de services. L'utilisation de faux comptes est possible. Les risques encourus par les contrefacteurs sont limités car ils agissent souvent d'un cybercafé à partir de plusieurs ordinateurs. Leur fraude consiste à poster des avis très positifs pour leurs clients et des commentaires de dénigrement pour les concurrents. Une autre technique consiste en l'achat massif de 500 ou de 1000 faux avis auprès de sociétés en ligne, afin de placer un film en tête ou en queue dans les sondages.

\subsubsection{La modération est biaisée, les sites ne conservant que les avis qui lui sont favorables tout en retardant la diffusion des avis négatifs}

Le traitement différencié consiste à ne publier que des avis clairement positifs et à supprimer la totalité ou une partie des avis négatifs. La tromperie consiste aussi à différer la diffusion des avis négatifs ou à assurer une «médiation » par le site auprès des contributeurs défavorables au produit pour faire disparaître les avis négatifs.

\subsubsection{La rédaction de faux avis par des salariés de l'entreprise ou un prestataire extérieur}

Les commentaires sont en fait des publicités déguisées que l'entreprise elle-même ou le gestionnaire du site poste sur le site. Des salariés de l'entreprise peuvent aussi être sollicités pour rédiger des avis sur des sites dont ils sont clients, d'où des conflits d'intérêts. En contrepartie, ils perçoivent par exemple un gain à un jeu concours interne. Ces avis sont ensuite mélangés à de vrais avis de consommateurs. Les faux blogs de cinéma appelés des flogs, néologisme construit à partir de fake et de 
blog ont par ailleurs pour principe de fonctionnement de diffuser des faux avis pour le compte d'une entreprise.

\subsubsection{Billets et étoiles sponsorisés}

Les places de cinéma sont remboursées et parfois accompagnées d'une rémunération, en échange d'un commentaire de film pour lequel l'internaute a été missionné par un site commercial. Le site controversé http:// www.CeSoirCiné.fr qui fonctionnait sur ce principe a été fermé en 2012. Les contributeurs ne peuvent pas attribuer un avis négatif à un film pour lequel ils ont reçu une mission par le gestionnaire du site. Des sites de films payent aussi des étoiles pour attribuer le meilleur score possible à un film.

Par ailleurs, les sources d'énonciation possibles de ces contrefaçons relèvent de six grands types : gestionnaires de sites créant de faux avis pour promouvoir un nouveau film, prestataires extérieurs qui agit contre rémunération, agences de publicité ou services de marketing, attachés de presse, employés de studios de production ou diffuseurs de cinéma.

Les suites contentieuses données aux contrôles relèvent de l'article 20 de la Loi sur la Confiance dans l'Economie Numérique (LCEN) du 21 juin 2004 et de l'article L121-1 du Code la consommation. La loi Chatel pour le développement de la concurrence au service des consommateurs du 3 janvier 2008 renforce les sanctions prévues par le Code de la consommation. Cette loi précise que les pratiques commerciales trompeuses reposent sur des allégations, indications ou présentations fausses ou de nature à induire en erreur sur les caractéristiques du service ou du bien. Elle condamne en outre le fait de dénigrer les concurrents, de dissimuler une information substantielle ou de ne pas indiquer sa véritable intention commerciale, de ne pas respecter les conditions de l'agrément, de l'approbation ou de l'autorisation reçue, d'utiliser un contenu promotionnel dans les médias sur un produit ou d'un service financé par le professionnel lui-même, sans l'indiquer clairement dans le contenu. Il faut ajouter que la Directive européenne $n^{\circ} 2005 / 29$ recense pas moins de vingt et une pratiques commerciales déloyales.

Nous proposons d'utiliser ces éléments juridiques et économiques pour analyser les commentaires et leurs sources d'énonciation puis leur environnement sur le Web. L' échantillon d'avis choisis de façon aléatoire est extrait de sites de commentaires en ligne.

\section{Analyse contextualisée d'un corpus de commentaires}

Notre corpus est constitué de commentaires relatifs au film Jobs (2013) réalisé par Joshua Michael Stern en 2013, avec Ashton Kutcher dans le rôle principal. Ce film, présenté comme un drame et un film biographique (un biopic en anglais), porte sur la vie du fondateur d'Apple, décédé en 2013. Les sites de commentaires de cinéma qui ont couvert ce film sont nombreux. Citons en particulier les sites Web Avisrumuneres, SensCritique, L'internaute, le passeur critique et Allociné. Ce dernier site est considéré d'après des cinéphiles interrogés comme l'un des plus fiables. Cependant, il n'est pas situé en dehors des logiques commerciales. Il dépend pour $98 \%$ du capital de la société française Fimalac depuis juillet 2013. Cette entreprise française investit dans les domaines de la finance, de l'hôtellerie, du tourisme, de l'immobilier, de la mode, de la gastronomie, des jeux vidéos et du secteur du numérique.

\subsection{Analyse d'identités virtuelles}

L'activité intense de cinéphiles passionnés est l'une des caractéristiques majeures des sites de commentaires amateurs de cinéma. Les instances d'énonciation et les identités virtuelles méritent un traitement particulier.Les commentaires filmiques de Phil Siné, pour ne citer que cet internaute, dans un temps sur son blog, puis dans Leplus sur le site du Nouvel observateur et surtout sur les sites de cinéma, constituent une contribution fort intéressante au Web participatif tout comme les sélections 
musicales du même contributeur sur sa chaîne You Tube. Est-il si important par ailleurs de savoir qu'à chaque fois qu'une personne clique sur les publicités des blogs, le propriétaire du site peut percevoir une rémunération? L'essentiel est de rester libre et loyal dans ses commentaires, comme le prévoient les chartes des usagers. Nous avons donc choisi d'analyser trois identités virtuelles d'internautes, issues d'Allociné et de SensCritique. Leur choix s'explique par le fait qu'ils font partie des contributeurs de cinéma parmi les plus prolifiques. La question qui va se poser est de savoir comment sont structurées les identités virtuelles et quels liens existent entre ces identités et d'autres sites Web.

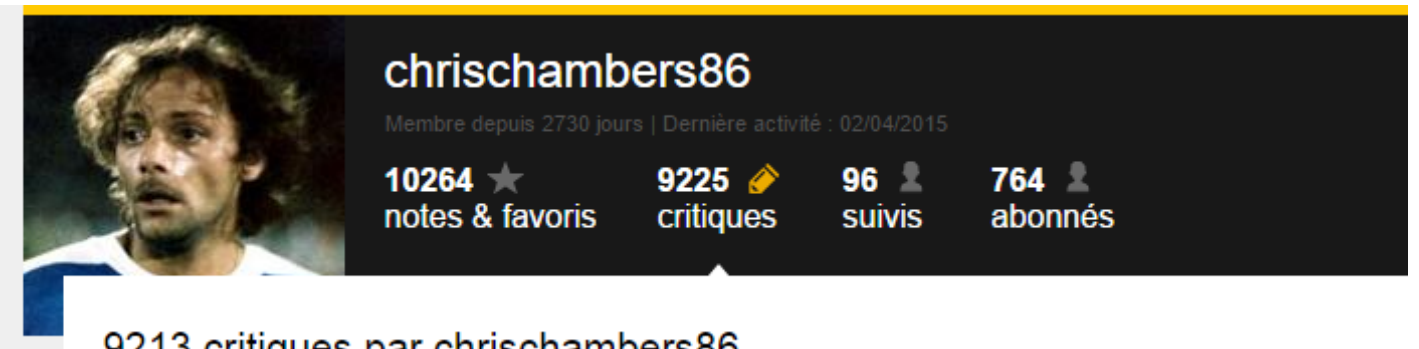

9213 critiques par chrischambers 86

\section{Interprétation}

Le signe visuel choisi par ce contributeur d'Allociné est provocateur : il renvoie à une icône du cinéma français, Patrick Dewaere, qui interprète dans Coup de tête de Jean-Jacques Annaud le rôle d'un footballeur difficile à manager. L'image détournée se retrouve aisément en saisissant le nom du film sur Google. Autre provocation : le pseudonyme choisi, chrischambers, qui renvoie à une icône du football américain, le point commun entre le pseudonyme et la photographie étant les sports collectifs. Cet exemple nous oriente vers le détournement d'images et de patronymes de célébrités. Cette pratique récurrente sur le Web peut poser problème si l'image est protégée ou s'il s'agit d'un nom de marques de vêtements de sports ou encore si le nom emprunté d'une personne vivante ou décédée est associé de façon malveillance à un compte.

En tant que contributeur passionné, chrischambers 86 avait posté 9213 critiques sur le site d'Allociné en seulement 2730 jours, au 2 avril 2015. Rien n'indique pour autant l'existence d'une pratique déloyale car il peut s'agir aussi de commentaires antérieurs à son inscription sur le site. Une critique peut aussi tenir en trois lignes et se rédiger en quelques minutes. Rien ne permet de savoir non plus s'il agit seul ou pour le compte du site en tant que prestataire de service. Chrischambers86 était suivi par 764 abonnés en 2015 et par 1031 abonnés en 2017, ce qui fait de lui un leader d'opinion. Sa collection de 1260 films est directement accessible sur le site, sans que l'on sache exactement le contrat qui le lie à Allociné.
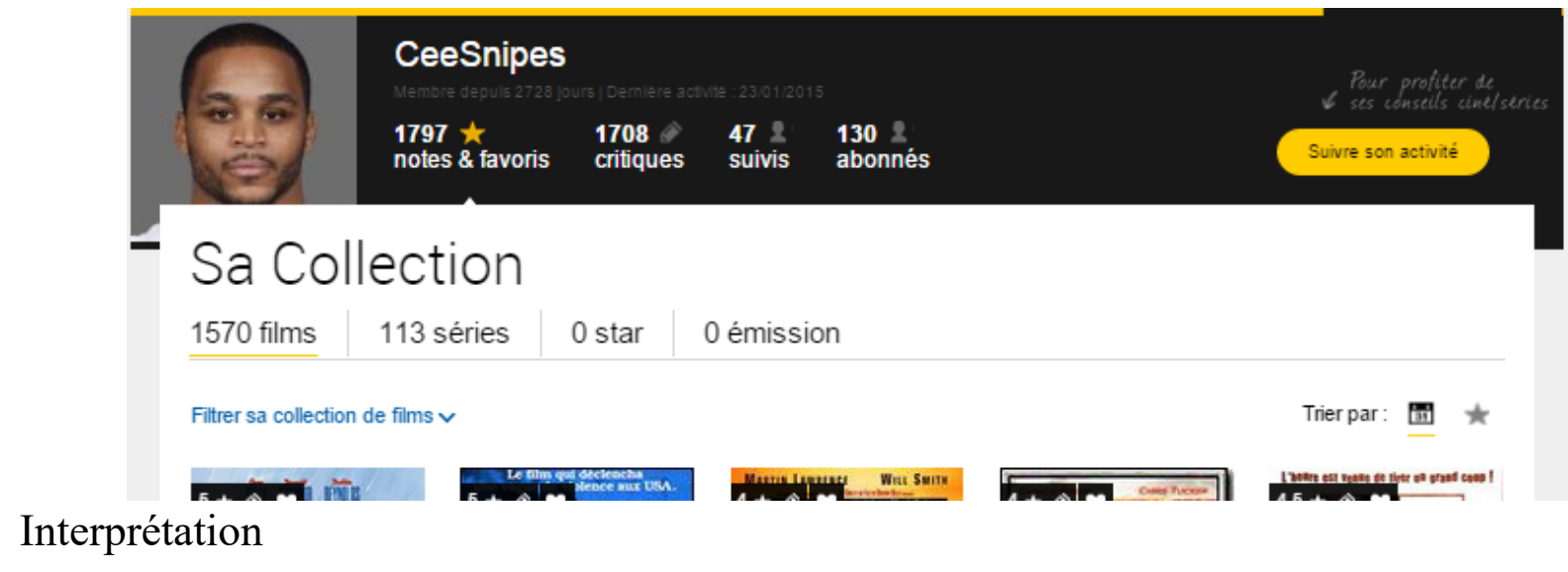

Le pseudonyme CeeSnipes est à attribuer, après recherche sur la toile, à un étudiant. Son identité réelle se retrouve aisément sur le Web. Ce cinéphile est un critique très actif à la fois sur Allociné, Senscritique et sur son blog Le critiqueur fou, en fonctionnement depuis 2011. Il commente chaque année plusieurs centaines de films selon un mode de présentation immuable : fiche technique, affiche, synopsis, critiques et notes. Ses nominations, les Ceesnipes Awards 2012, des meilleurs films et 
acteurs de l'année attestent d'une expertise incontestable. Il se présente aussi comme un leader d'opinion, plus exactement un « influenceur». Sur le seul site Allociné, il avait déposé 1797 notes et 1708 commentaires en 2015 et mi 2017, il a présenté 1793 critiques et 1351 notes. Le nombre de ses abonnés a progressé, 130 en 2015,158 en 2017. Des interrogations subsistent cependant: le gestionnaire d'Allociné présente son commentaire du film Jobs comme «la critique positive, la plus utile », ce qui peut laisser penser à une contribution sponsorisée par le site ou à une publicité déguisée. Les notes attribuées par ce cinéphile passionné se situent pratiquement toutes entre $6 / 10$ et 10/10, ce qui ne peut se concevoir que s'il a choisi de ne traiter que des films qui lui ont plu. Comme dans le cas précédent, il est donc difficile de savoir si ce contributeur est sponsorisé par le site.

www.Senscritique.com

Pseudo : MC4815162342 .159 abonnés | Lire ses 1479 critiques

4 - Très bien

« Jobs est un film fabuleux, Kutcher dans le rôle du célèbre inventeur est incroyable, le reste du casting est tout aussi bon, les décors sont géniaux, la reconstitution est fabuleuse, une histoire que tout le monde ne connait pas, moi personnellement je ne la connaissais pas et c'est génial de découvrir l'histoire de Steve Jobs, le film aurait cependant mérité d'être plus long pour bénéficier de plus de détail mais il reste splendide $»$.

\section{Interprétation}

Cet énonciateur compte 1479 critiques et rassemble une communauté de 160 abonnés. Le pseudonyme choisi - MC481562342- est surprenant. Une recherche sur la toile permet de retrouver ce pseudonyme associé à un site commercial de doudounes ou de gilets de la marque Moncler (www.optimosystems.cotiza/fr/chaussure-air-max-femme-933306.asp) et à un site de jeux (www.xboxlivescore.com)! Il est difficile cependant d'établir une cohérence entre tous ces sites, sauf à considérer que le contributeur mène peut-être plusieurs activités commerciales de front et qu'il traite en priorité des jeux qui sont des adaptations de films ou vice versa. Et surtout, le commentaire parait étrangement dithyrambique et vague. Le film est " fabuleux », «splendide », l'interprétation est « incroyable », les décors " géniaux », la reconstitution «fabuleuse », enfin il est " génial »de découvrir l'histoire. Il s'agit typiquement du type de commentaires pour lequel il est douteux que l'énonciateur ait vu le film, se contentant ici d'user et d'abuser d'hyperboles.

\subsection{Les écrits conversationnels en quasi-direct}

Les sites de commentaires de cinéma diffusent de très nombreux « écrits conversationnels ». Il s'agit de formats courts, incisifs, spontanés, volontiers ironiques ou caustiques, parfois expéditifs, dans l'esprit des forums de discussion (Anis, 1998. Marcoccia, 2000).Pour Anis (1998, p164), ces communications en quasi-direct, expriment des émotions et des pulsions et constituent un "écrit conversationnel », proche d'un oral spontané.

1-Margauxperso. 5/ 5.22 likes contre 21 non likes. A noté 174 films. www.Allociné.fr

«Un film passionnant! Cet homme au destin hors du commun a su croire en son idée révolutionnaire, et devient à mes yeux le génie le plus charismatique de sa génération. Ce film donne de l'espoir à tous les jeunes créateurs d'entreprise et à ceux qui souhaitent se battre pour le projet. Je recommande à $3000 \%$. » 
Interprétation

L'incitation à aller voir le film se fait pressante : « je recommande le film à $3000 \%$ ». Il est surtout écrit dans la même veine et le même format (quatre lignes) que les autres commentaires du même énonciateur sur le site. A chaque occasion, les films sont recommandés, ce qui peut paraitre suspect, sauf à considérer que ce contributeur ait choisi de ne traiter que les films qui lui ont plu.

2-Antoine G. : 4,5/5. Excellent. Date :24 août 2013 www.Allociné.fr

«Un bon biopic. Les acteurs nickel mais on ne voit pas assez sa vie personnelle à mon avis/ réalisation parfaite à voir pour les fans de Jobs ».

3- Pseudo : Sebabarre .Note : 6/10 www.senscritique.com

« C'est pas hyper bien joué, et le film est quand même hyper à charge. Pis ça reste un biopic ».

Interprétations

Le premier commentaire parait authentique. Les écarts sémio-linguistiques apportent des indices concordants en ce sens. Cependant, le problème qui se pose est de trouver une preuve suffisamment pertinente pour ne pas s'en remettre à de simples indices. Il nous faut pour cette raison utiliser la courbe de Gauss, appliquée à l'exemple suivant.

Le second commentaire, incisif, est très proche aussi de l'écrit conversationnel propre aux forums (Anis,2000). L'énonciateur, Sebabarre, a-t- il produit un commentaire vrai ? Ce contributeur est toujours en activité sur SensCritique en 2017. Une recherche sur le Web permet de trouver aisément son identité réelle. La courbe de ses notes pour les jeux, musiques et films qu'il a critiqués sur le site est similaire à la fonction de densité d'une loi normale en probabilités, soit la courbe de Gauss. Ce commentaire est donc mathématiquement non contestable. Autrement dit, il s'agit très probablement d'un commentaire authentique.

4-Spiderbaby (619 critiques). 0, 5/5 Date : 20 décembre 2013 www.Allociné.fr

«Euh... A quand un biopic sur Pinault, Bolloré ou sur le patron de Leclerc ?»

Une recherche sur le Web permet de mieux cerner ce contributeur qui emprunte son pseudonyme à un film d'épouvante de Jack Hill sorti en 1964. Spiderbaby est l'un des commentateurs les plus percutants sur Allociné et SensCritique. Sa contribution des 22 films les plus détestables de l'histoire est particulièrement corrosive. Le film Jobs est critiqué dans le même ton que sur Allociné : «Euh... A quand un biopic sur le patron de Carrefour ». Ces commentaires paraissent authentiques. On conçoit mal ce contributeur percevoir ponctuellement une rémunération par un donneur d'ordre pour critiquer les films d'un distributeur concurrent.

5-Jrmy Bodin (131 critiques) 6 janvier 2015. 1 étoile/10 www.senscritique.com

Titre : Ahh§ Enfaite Steve est mort pour ne pas voir ce film

Critique : « ce film est réalisé par un tel Joshua truc, réalisateur inconnu quoi a une filmographie à chier... «Jobs »commence par une intro assez spéciale, ou l'on voit Steve présenter dans une keynote (la seule du film).l'Iphone ? Non, l'Ipod. Cette séquence avait en fait pour but de montrer le magnifique travail de maquillage qu'il faut faire pour qu'Arthur Kutsler devienne teve Jobs, juste sa...A ... ». 
Ce commentaire corrosif est très certainement un vrai commentaire en raison des nombreux écarts sémio-linguistiques qu'il comporte et de sa tonalité corrosive, en phase avec la culture des sites participatifs.

\subsection{Les formats longs apparentés au communiqué de presse ou à l'annonce publicitaire}

Les formats longs dans les rubriques de commentaires amateurs ne relèvent pas de la réaction impulsive. Il s'agit de communications en différé, très travaillées, pouvant s'étendre sur une ou deux page(s) écran(s). Ils peuvent s'apparenter aux genres très codifiés du communiqué de presse ou de l'annonce publicitaire. On peut soupçonner le travail d'un attaché de presse et d'une agence de marketing qui auraient placé leur commentaire dans une rubrique dédiée aux contributeurs amateurs.

1 - Loren C (Allociné) 26 août 2013 .Réponses aux commentaires : 8 likes contre 11 non likes.14 abonnés | Lire sa critique | Suivre son activité 5 - Chef d'oeuvre

«Jobs, certains diront que ce film est bâclé, qu'il ne va pas au fond des choses, d'autres affirmeront que la performance d'acteur de Ashton Kutcher est fausse, surjouée, voire grotesque et d'autres crieront haut et fort que ce long métrage est fade, ennuyeux et sans grand intérêt. Et cela est humain. Seulement, je ne peux m'empêcher de penser : sont-ils insensibles, ou tout simplement trop bêtes pour s'apercevoir que Jobs est un chef-d'oeuvre ? Certes, tous se plaindront du caractère minimaliste de ce film, le fait qu'il n'ai pas retracé toute la vie de ce fabuleux génie; n'ont-ils juste pas pensé, cela ne leur est-il jamais venu à l'esprit que tout est là, dans ce film ? Que tout ce qui vient après n'est que la continuité du génie de Steve Jobs? Que cela n'est que détail ? Que ce qui est le plus important, le plus passionnant et le plus captivant se concentre dans ce long métrage ? Il a déjà fait ses preuves, il n'a plus rien à prouver. La scène première du film, la présentation du tout premier Ipod n'est que le commencement de la continuité. Tout ce qui vient après n'est que sommaire. Et ce film, je le défendrai quoi qu'il arrive, car il retrace parfaitement ce qu'est et sera toujours Jobs : un visionnaire, un génie en son genre, un fou. Un fou qui a su changer les choses dans notre société, nous rendre meilleurs et ne faire qu'un avec la technologie. Je tiens aussi à ajouter que la performance d'Ashton Kutcher, est tout bonnement impressionnante; l'impression de voir défiler Steve Jobs en chair et en os sur le grand écran m'a laissée bouche-bée. Il l'interprète, se l'approprie, l'incarne avec tant de réel que cela en est extraordinaire. Nous sommes bien loin de tous ses rôles précédents, est ce rôle ci, est pour moi son meilleur rôle. Nous avons pu entrer au plus profond de son être, de son génie, comme si nous étions, là, dans sa tête. Jobs, m'a bouleversée d'une manière que je ne saurai décrire, il m'a ouvert les yeux; la vie est impitoyable, et quand vous rêvez secrètement de changer le monde, la meilleure façon d'y arriver est la détermination de se dire que, oui je suis fou, mais ce sont les fous qui s'imaginent qu'ils peuvent changer le monde. Pour conclure, je dirai que Jobs n'a pas été aussi bien accueilli qu'il le méritait. Je pense que, la plupart des personnes, malheureusement n'ont rien compris, vous n'avez rien compris au génie, vous n'avez rien compris du tout. Car, pour dire que ce film est raté, inutile, bâclé et j'en passe, vous ne devez réellement pas, ne serait qu'une seule seconde, su comprendre son intérêt et quel chef-d'oeuvre il était. Et c'est tellement désolant. Je finirai ce point de vue par une citation du film, une sorte de message à vous qui allez désapprouver cette critique, et qui allez gentiment cliquer sur le smiley rouge, car oui, c'est vrai, vous êtes ignorants et incapables de reconnaître un vrai chef d'oeuvre." Here's to the crazy ones, the misfits, the rebels, the troublemakers, the round pegs in the square holes... the ones who see things differently -- they're not fond of rules... You can quote them, disagree with them, glorify or vilify them, but the only thing you can't do is ignore them because they change things... they push the human race forward, and while some may see them as the crazy 
ones, we see genius, because the ones who are crazy enough to think that they can change the world, are the ones who do. »

Ajoutée le lundi 26 août 2013

Interprétation

L'énonciateur n'a curieusement produit qu'un seul commentaire, dupliqué en outre sur un site de streaming, ce qui pour la DGCCRF ferait partie des méthodes employées dans les publicités déguisées. Son pseudonyme n'a pas été retrouvé sur les sites web ou les réseaux sociaux.

Les techniques de persuasion et de manipulation sont largement employées. Elles ne portent pas sur le film, qui est à peine évoqué, mais sur les affects, la recherche d'une complicité de type émotionnel. Ce commentaire se présente en effet sous la forme d'un plaidoyer en faveur du film (5 étoiles, soit la catégorie chef d'œuvre).L'énonciateur cherche à créer tour à tour une connivence avec les internautes en utilisant des mécanismes psychologiques d'adhésion avant de leur refuser toute capacité de discernement, les qualifiant même d' «ignorants». Il termine par une citation de 516 signes en anglais (le film est pourtant sorti avec un doublage en français) qui vise à atteindre, à nouveau, chez l'internaute influençable l'estime de soi. Il conviendrait donc d'adhérer pleinement au film ou alors d'entrer dans la catégorie des internautes incultes. La tentative de manipulation des affects a échoué puisque cette longue critique de 3449 caractères n'a suscité que 8 likes. 


\begin{abstract}
3.5- Bien
The Social Network s'était intéressé à Facebook@ en 2010 et avait confié le rôle de Mark Zuckerberg à Jesse Eisenberg. Owen Wilson et Vince Vaughn durent jouer Les Stagiaires chez Google@, Benedict Cumberbatch interprétera dans Le Cinquième Pouvoir Julian Assange, fondateur de Wikileaks 8 tandis que Jobs explore la piste de lentreprise la plus valorisée au monde. Applee, et retrace une partie de la carrière de son créateur, le regretté Steve. Ainsi, après avoir pris le contrôle de nos modes de vie, voilà que le marché du hightech se greffe une place au septieme art et joue la carte biopic pour monter en puissance. Alors, Ashton Kutcher sera-t-il à la hauteur du personnage quili interprète ? Cest sur une introduction qui sera également la dernière image du personnage, que commence le film, alors que Steve Jobs, The CEO of the company, nous présente sa dernière création, riPode, dans une scène exploitant au mieux la force marketing d'Apple\&, arguant chiffres et citations dignes de la pomme. Une scène véhiculant émotion et frissons, après seulement quelques minutes de pellicule. Cette intensité, dailleurs, qui nous sera à nouveau communiquée via une publicité pour le Macintosh@, renforce la dynamique du film et réalise, soit dit en passant, un exploit en nous arrachant ledit sentiment si rapidement. Dissimulé sous un trés beau maquillage, Ashton Kutcher se complait dans son róle, adopte la méme démarche, nage dans les mêmes vêtements et nous regarde comme lui le faisait. De mêne que dans L'Effet Papillon, Tacteur malencontreusement trop embourbé dans ses róles comiques bat des ailes et accapare le grand écran, alors que les apparitions de James Woods, Kevin Dunn ou Dermot Mulroney, bien que surprenantes, se montrent presque anecdotiques. Certes, le film met en avant un homme sưr de lui, prenant les gens de haut, n'en faisant qu'à sa tête et vouant sa vie à ses créations, mais il enseigne aussi la culture de fentreprise et explore les débuts de rére purement informatique, en abordant la création du casse-briques, la guerre AppleB/IBM8, les fondations de la marque à la pomme ou encore fanimosité nais sante qu'elle a eu avec Microsofte. Mais, par-dessus tous ces artifices, c'est un film indépendant et simple, sans armada, qui raconte comment un homme a su (et pu) révolutionner lélectronique d'aujourd hui, dans une composition claire et agréable du mythe qui accompagne rHomme. Le principal reproche qui peut lui étre fait reste ses prises de position dans les axes qu'il développe. Ainsi, la participation de Steve Jobs dans Pixare est complètement obscurcie, la rivalité et les procès avec Microsoftes sont à peine mentionnés tandis que ses problèmes de santé ne figurent méme pas à rordre du jour ! Difficile de comprendre du coup pourquoi le film dure plus de deux heures. Car, bien que le film soit loin d'etre mauvais, deux heures dix. c'est relativement long. Surtout quand Thistoire ne tire que trop davantage sur le roble du conseil d'administration et son influence sur la quête de finnovation. De mẻme, pourquol s'arréter en 2001 avec la présentation de riPode, alors que riPhone8, riPade ou les MacBook \& suivront ? On peut alors penser que le film voulait explorer les prémices d'Appleق. ses déboires et sa reconquête du marché. Mais pourquoi ne pas interagir avec ce qui fit réellement connaitre Steve Jobs aux générations actuelles ? Cela aurait été roccasion d'explorer d'autres pistes et de catapulter Apple@ dans sa querelle judiciaire avec Samsung@e, tout en citant rinfluence de Google@. Là sont les limites du métrage, qui se veut plus terre-d̀-terre et moins tourné sur la raison sociale que sur Thomme qui la façonnée. Une direction somme toute honorable. En se cachant sous les traits de Zuck". Jesse Eisenberg a permis à The Social Network de remporter trois Oscars. Pour interpréter Steve Jobs. Ashton Kutcher s'en tire honorablement, mais il y fort à parier que le film sera absent des Acadenty Award, en raison du manque de communication de réquipe pour le film. Fait ironique, quand on sait qu'Apple@ a dépensé $1,000,000,000$ s en publicité en 2012.

Aloulee le leudi 5 septembre 2013
\end{abstract}

\title{
Interprétation
}

Dans le florilège des images présentes sur Allociné, cette photographie remplit une fonction d'icône en représentant l'une des figures les plus symboliques du $7^{\circ}$ art. Une rapide recherche sur le Web permet d'attribuer la paternité de ce cliché de Charlie Chaplin à la General photographic Agency/ Getty images. L'articulation de cette image au commentaire est productrice de sens car il s'agit de situer les commentaires dans une filiation, celle de la célébration du $7^{\circ}$ art. Une autre relation intertextuelle apparait dans l'allusion au film The Social Network, premier film à thèse sur la « révolution » sociale engendrée par Facebook.

Par ailleurs, le profil de ce cinéphile qui se présente lui-même comme un "passionné » est aisé à retrouver sur le Web. Sa collection de films sur le site d'Allociné, riche de 1100 films, lui confère une expertise certaine. Quant à la découverte de son patronyme, elle nécessite une fouille approfondie. Le discours mis en ligne présente des similitudes troublantes avec le genre codifié du communiqué de 
presse. En outre, chaque marque citée est suivie du symbole copyright, ce qui est un peu insolite pour un commentaire. Enfin, la chute est une charge contre le manque d'investissements en communication publicitaire de l'équipe de production. Il est difficile pourtant de conclure que ce cinéphile passionné soit sponsorisé.

3-Pseudo :Ionnaties . www.Télérama.fr et www.Allociné.fr le 9 septembre 2013

Note 4/5. Nombre de commentaires postés par l'énonciateur : 3

Commentaire :

La vie des grands hommes et femmes qui ont changé notre vie doit être connue au grand public. Leurs efforts et leur contribution au progrès de nos civilisations doit rester comme référence qui nous permettra de lutter contre la routine et contre le conservatisme qui s'oppose à toute forme de rêve et de changement. En plus, ce dernier peut être dangereux car l'immobilisme peut bien annuler toutes les luttes des peuples contre une société réactionnaire absurde illogique et despotique. Le cinéma a toujours été un art qui a animé les grands événements historiques ou la vie de ces personnages qui ne doit pas être ignorée. En effet le film Jobs nous fait découvrir la vie de Steve Jobs, qui, grâce à son anticonformisme, il est parvenu à s'opposer à l'IBM et de concevoir l'informatique comme un outil qui épanouit l'individu et développe sa personnalité libre. Loin de concevoir l'ordinateur comme une machine d'un système totalitaire qui contrôlerait le caractère de l'homme, il a pensé à la création quasiment artistique qui enrichirait la culture de ce dernier. Le retour aux années 70 met en évidence une belle époque pendant laquelle chaque individu voyait son avenir avec un optimisme que le XXIe siècle l'ignore complètement. Les références culturelles de chaque époque sont présentées sans défaut dans ce beau film qui retrace la vie de ce personnage historique. Nous devons signaler également que le réalisateur Joshua Michael Stern a bien mis l'accent sur l'aspect artistique de la personnalité de Steve. En effet la sensibilité de ce personnage se heurtait avec la réalité du monde des finances qui voulaient que l'individu perde sa personnalité et qui fait partie d'un grand troupeau guidé par un chef. Cependant, nous devons souligner un défaut de ce film: La lenteur de certaines scènes qui pouvaient bien désorienter le spectateur. Le film Jobs constitue un témoignage vivant sur la personnalité de Steve et c'est cela qui nous permet de penser que le septième art est toujours vivant. C'est un outil qui nous permet de garder l'essence de notre existence menacée la civilisation du XXIe siècle qui sacrifie toute forme idéalisme au profit des intérêts des maisons des finances.

\section{Interprétation}

Ce commentaire de 2199 signes se caractérise par sa distance énonciative et la quasi-absence de la fonction émotive. Il est très proche dans ses caractéristiques lexicales d'un communiqué pour le moins soporifique. Il laisse apparaitre l'opposition (qui est la trame du film) entre le conservatisme de la société des années 1970 et l'anticonformisme de Jobs, incarnée par sa lutte contre IBM pour libérer l'homme par l'informatique. Le commentateur n'a publié que très peu d'avis et les avis de cette longueur sont rares. En outre, le même commentaire est intégralement présent sur deux sites concurrents, ce qui peut faire partie des techniques employées dans les publicités déguisées et la construction d'un média planning.

Pseudo : Gwimdor. Site SensCritique

"L'analogie est facile mais Jobs c'est ce qu'aurait pu être The Social Network si le projet n'avait pas été confié à quelqu'un d'aussi expérimenté que Fincher, en offrant à un scénariste random qui a bossé sur des projets médiocres et qui n'avait pas encore réalisé le moindre film à savoir, Joshua| 
Michael Stern, la production n'avait pas une ambition démesurée pour ce projet et ça ressent énormément à l'écran!

Pourtant c'est bien dommage, qu'on aime Apple ou pas, un biopic sur un homme visionnaire qui a toujours su s'entourer de personnes compétentes pour changer le monde à sa façon méritait un traitement plus pertinent approfondi, ici tout va trop vite ou pas assez ! Dans une réalisation fade semblable à un mauvais téléfilm, le récit se perd régulièrement dans un rythme absolument pas maîtrisé, s'attardant sur des détails insignifiants et soporifiques pour traverser les grandes étapes qui ont permis à Steve Jobs et Apple de devenir des figures incontournable des années 80 ! Tout va trop vite et pas assez, en 30 minutes le début de la réussite, l'explosion et la déchéance sont amenés sans finesse quand on s'attarde sur le baba cool marchant pieds nus à la fac. Ashton Kutcher dans le rôle titre offre une performance correcte sans briller pour autant, hormis peut-être lors de la scène d'ouverture dans laquelle Jobs annonce l'iPod et qui dans sa gestuelle et sa façon de s'exprimer capte assez bien l'aura que pouvait avoir le visionnaire dans ces moments-là. Moins efficace qu'un documentaire d'M6, ce long métrage n'a ni les qualités cinématographiques nécessaires pour offrir un biopic divertissant ni la substance pour relater la vie de Jobs correctement. On retiendra juste le génial Bob Dylan pour nous accompagner musicalement, la présence de J.K Simons (Schillinger dans Oz) toujours appréciable et quelques scènes réussies puis on oubliera vite l'expérience qui ne mérite pas un déplacement en salle. »

\section{Interprétation}

Ce discours de 1934 signes est beaucoup plus rythmé et chargé d'émotions que le précédent commentaire et il a été beaucoup moins travaillé dans la phase de relecture du texte. L'expression est marquée par cette légèreté dans l'expression qui caractérise les forums, à l'instar de l'allusion humoristique au « baba cool marchant pieds nus à la fac». Avec 4300 notes dont 2400 pour des films et seulement 309 critiques, il s'agit d'un utilisateur prolifique et passionné souhaitant « répertorier ses films cultes avant de perdre la mémoire ». Il est référencé sur plusieurs sites Web comme un amateur passionné par le cinéma et les jeux vidéo. Son nom, qui se rapproche de celui d'un Elfe, est emprunté à l'œuvre fantastique de Tolkien. Sa culture cinématographique apparait dans plusieurs relations intertextuelles relatives à des films et à des acteurs. Il s'agit incontestablement d'un commentaire authentique.

\section{Conclusion}

Les problèmes de contrefaçons nous semblent beaucoup plus relever de la responsabilité des platesformes ,de leurs outils informatiques de traitement des commentaires et des sources d'énonciation que des commentateurs eux-mêmes. Un traitement loyal des commentaires et la recherche des faux avis constituent une obligation pour les gestionnaires de ces plates formes.

Les commentaires choisis dans cet article ont été placés dans deux catégories opposées : des écrits conversationnels d'une part et de longs écrits apparentés à des genres comme le communiqué de presse ou l'annonce publicitaire d'autre part. Si les écarts nombreux vis-à-vis des normes sémio-linguistiques laissent accroire qu'il s'agit de formes sémiotiques authentiques, c'est incontestablement la recherche sur d'autres sites qui permet de retrouver à partir d'un simple pseudonyme l'identité réelle de la plupart des contributeurs et dans bien des cas leurs activités professionnelles. Les détournements d'images d'icônes du $7^{\circ}$ art, de personnages ou de films célèbres sont récurrents parmi les internautes passionnés tout comme l'utilisation de patronymes connus de l'univers du cinéma voire du sport.

A contrario, il existe plusieurs formes de manipulation. L'absence du pseudonyme sur la toile ajoutée au fait que le même commentaire se retrouve sur plusieurs sites de presse laisse penser qu'il s'agit d'une publicité déguisée ou d'un communiqué de presse, déclinée sur plusieurs supports dans le 
cadre d'un média planning. Les commentaires trop généraux et hyperboliques font supposer que les films commentés n'ont pas été vus et qu'il s'agit de faire la promotion d'un film. Par ailleurs, un commentaire se caractérisant par une distance énonciative sied mal avec le commentaire critique d'un cinéphile passionné comme le sont la plupart des contributeurs. A contrario, les écrits conversationnels analysés se caractérisent par leur caractère souvent corrosif voire même féroce.

Les cinéphiles passionnés disposent dans la plupart des cas d'un blog et sont suivis par des centaines d'abonnés. L'un d'entre eux se définit comme un influenceur. Le nombre de contributions postées oscillent quant à lui d'un commentaire unique à 9213. Ces deux extrémités paraissent douteuses, la première parce qu'il peut s'agir d'un communiqué de presse émis par un énonciateur à adresse éphémère, la seconde parce qu'il peut s'agir de l'expression d'une activité professionnelle. Une question cruciale concerne les relations contractuelles entre les contributeurs passionnés et le site de cinéma qui héberge leur collection de 1000 films et plus. Ces commentateurs passionnés sont -ils des prestataires de service qui commentent les films pour la plate-forme?

A partir des énoncés étudiés, il est difficile de souscrire à l'hypothèse que les sites de cinéma subissent autant de contrefaçons que les sites de e commerce. L'utilisation de la courbe de Gauss nous est apparue comme l'une des méthodes les plus efficaces pour évaluer en cas de doute l'ensemble des contributions d'un commentateur.

On peut raisonnablement penser que des communiqués de presse ou des annonces publicitaires conçus pour des sites professionnels ont été postés dans des rubriques amateurs pour tenter d'influencer le choix des internautes. Pour la DGCCRF, des attachés de presse ou des agences de marketing peuvent tenter de se glisser dans les rubriques qui ne sont pas les leurs. Seul, le principe de contrôles aléatoires exercés par la DGCCRF pourrait clarifier une situation incertaine. Mais, quels que soient les véritables pourcentages, l'essentiel est de noter que les contrefaçons faussent le jugement des cinéphiles dans des proportions variables.

On pourrait établir une gradation de ces faux avis et de ces fausses notes. A un premier degré, nous placerions le faux avis posté occasionnellement par une personne physique qui agit seul. A un second degré, la personne physique ou morale qui organise méthodiquement la diffusion de faux commentaires pour discréditer les concurrents et/ou valoriser ses clients. A un troisième degré, les votes diffusés automatiquement par de faux comptes obtenus à partir de sites pirates qui captent aussi un pourcentage sur les votes publiés. A un quatrième degré, l'utilisation de petits programmes (ou robots) qui permettent de capter des comptes à l'insu de leurs propriétaires.

Le nombre des faux commentaires semble ne pas avoir augmenté récemment, d'après les enquêtes menées par la DGCCFR en 2014 puis 2016 alors qu'ils avaient augmenté brutalement de 45\% entre 2012 et 2013. La délivrance d'un certificat AFNOR depuis septembre 2013 a sans doute permis de réduire le nombre des faux avis. L'AFNOR délivre en effet un certificat pour les sites Web capables de démontrer la fiabilité de leur traitement de commentaires. Il s'agit d'une amélioration même si cette norme n'a aucune valeur juridique contraignante.

Toutes ces considérations auraient enfin parfaitement leur place dans une éducation critique aux médias numériques.

\section{Webographie}

http://www.economie.gouv.fr/dgccrf/consommateur.asso-par-secteur/e-commerce/faux-avis-de-consommateur-surinternet

https://www. Incapsula.com/blog/blog-traffic-report-2016.html

http://www.premiere.fr/cinema/News-cinema/twilight-hunger-games-balnche-neige-le-marketing-va-t-il-trop-loin $24 / 11 / 2011$ 
Bernard Stiegler : http://www.latribune.fr/actualites/economie/France/20090723trib000402565/bernard-stiegler-leconsumérisme-a-atteint-ses-limites

\section{Bibliographie}

Olivier Andrieu. Réussir son référencement Web. Ed.Eyrolles. Paris. 2015

Jacques Anis. Texte et ordinateur, l'écriture réinventée. Ed. De Boeck Université. Bruxelles. 1998

R. Barthes. «Le message photographique » in l'Obvie et l'Obtus, Essais critiques III. Le Seuil. Paris. 1982

Guillaume Calafat, Arnaud Fossier, Pierre Thévenin. Droit et sciences sociales: les espaces d'un rapprochement (éditorial). Ed. Tracés 27, 2014

Bruno Hénocque. Appropriation des messageries électroniques dans les entreprises en réseau. Presses universitaires de Bordeaux. Pessac. 2002.

François Jost. 50 fiches pour comprendre les médias. Ed.Bréal. Paris. 2010

Olivier Le Deuff. La formation aux cultures numériques. Edition FYP. Limoges.2012.

Agathe Lepage (sous la direction de). L'opinion publique. Presage. Ed. Dalloz.2006

Edgar Morin, Jean-Louis Lemoigne. L’intelligence de la complexité. Ed. l'Harmattan. Paris. 1999.

Roger Odin. Les espaces de communication .Introduction à la sémio-pragmatique. Collection «la communication en plus ». PUG. 2011.

Jean-Louis Lemoigne. La modélisation des systèmes complexes .Ed. Dunod-Bordas. Paris.1990.

Michel Marccocia . La représentation du non verbal dans la communication écrite médiatisée par ordinateur. Ed. Communication et organisation $n^{\circ}$ 18.Pessac. 2000

Jean-Pierre Teyssier. Frapper sans heurter. Quelle éthique pour la publicité? Ed. Armand Colin.Paris.2004. 